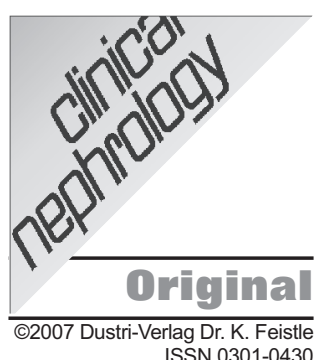

ISSN 0301-0430

\section{Key words}

fetuin-A - inflammation - left ventricular hypertrophy - chronic kidney disease - cardiovascular disease

\title{
Association between biomarkers of inflammation and left ventricular hypertrophy in moderate chronic kidney disease
}

\author{
S. Cottone, E. Nardi, G. Mulè, A. Vadalà, M.C. Lorito, R. Riccobene, A. Palermo, \\ R. Arsena, M. Guarneri and G. Cerasola
}

Dipartimento di Medicina Interna, Malattie Cardiovascolari e NefroUrologiche, Divisione di Medicina Interna, Nefrologia ed Ipertensione ed Unità Operativa semplice di Malattie Renali ed Ipertensione, Università di Palermo, Italy

\begin{abstract}
Aims: Left ventricular hypertrophy (LVH) is a predictor for cardiovascular mortality, and it is considered to be a surrogate marker of preclinical cardiovascular disease. This study aimed at evaluating whether fetuin-A plasma levels are decreased in patients with moderate chronic kidney disease (CKD) and their linkage to plasma concentrations of hs-C-reactive protein (CRP), cardiotrophyn-1 (CT-1), tumor necrosis factor- $\alpha$ (TNF- $\alpha$ ), propeptide of collagen Type I (PIP) and to LVH. Material and methods: We enrolled 64 moderate CKD and 55 essential hypertensives $(\mathrm{EH})$ with normal renal function as controls. All the patients underwent an echocardiographic examination; plasma samples were obtained to measure routine clinical parameters and the molecules listed above (measured by ELISA). Results: Among CKD there were 30/64 patients with $\mathrm{LVH}$, and in EH group 14/55 subjects had LVH. Fetuin A was reduced in CKD when compared with EH $(\mathrm{p}<0.0001)$. The comparison between CKD having LVH with those without LVH showed significant differences in plasma levels of fetuin-A $(p<0.002)$, TNF- $\alpha(p<0.01)$ and hs-CRP ( $\mathrm{p}<0.001)$, CT-1 and PIP ( $\mathrm{p}<$ $0.002)$. CKD with LVH had lower values of fetuin-A $(p<0.001)$, and higher values of hs-CRP $(\mathrm{p}<0.001)$ TNF- $\alpha(\mathrm{p}<0.001)$, CT-1 $(\mathrm{p}<0.001)$ and PIP $(\mathrm{p}<0.001)$ than EH with LVH. The multivariate analysis of correlation demonstrated that in CKD patients hs-CRP $(\beta$ $0.42, \mathrm{p}<0.00006)$, and systolic blood pressure $(\beta 0.29, p<0.02)$ were independent predictors of LV mass index. The relationship between LV mass index and fetuin-A did not reach statistical significance. Conclusions: For the first time in moderate CKD patients, we demonstrate that fetuin-A is decreased and relates to LVH depending on C-reactive protein.
\end{abstract}

\section{Introduction}

Patients with end-stage renal disease as well as hypertensives with normal renal function are at high risk for vascular atherosclerosis and left ventricular hypertrophy (LVH) [Chertow et al. 2004, Levy et al. 1990]. In turn, LVH is an independent predictor for cardiovascular morbidity and mortality, and it is considered to be a surrogate marker of preclinical cardiovascular disease [Frohlich et al. 1992, Mancini et al. 2004].

Nevertheless, limited data exist on determinants of LVH in patients in the earliest stages of CKD [Astor et al. 2004].

Fetuin-A, also called $\alpha_{2}$ Heremans-Schmid glycoprotein (AHSG), is a major circulating protein that is mostly synthesized in the liver and inhibits calcification by binding hydroxyapatite structures. Fetuin-A is ubiquitously present in the extracellular space at serum concentration of $0.5-1.0 \mathrm{~g} / 1$ [Ketteler et al. 2003].

C-reactive protein, a sensitive marker of inflammation, is thought to represent the state of chronic low-grade inflammation of the arterial vessel wall at atherosclerotic sites. C-reactive protein (CRP) predicts consistently cardiovascular outcome [Blake et al. 2003, Ross 1999].

Increased amounts of CRP were demonstrated to be associated with low concentrations of the glycoprotein fetuin-A and with enhanced cardiovascular and all-cause mortality in uremia [Ketteler et al. 2003]. These data may account for accelerated atherosclerosis.

Tumor necrosis factor- $\alpha$ (TNF- $\alpha$ ) is essentially produced by monocytes and macro-

Via del Vespro 129, 90127 Palermo, Italy sancott@tin.it 
phages. It is endowed with the capability of inducing endothelial activation, and it is the strongest known paracrine activator of monocytes and macrophages [Deanfield et al. 2005]. Both TNF- $\alpha$ and CRP were found in considerable quantities in atherosclerotic lesions [Clausell et al. 1999, Waltenberger et al. 1998], and we demonstrated recently a relationship between these molecules in essential-hypertensive patients [Cottone et al. 2006].

TNF- $\alpha$ was shown to contribute to the remodeling process in the failing myocardium [Feldman et al. 2000].

Furthermore, it was shown that cardiotrophyn-1 (CT-1) [Gonzales et al. 2005], a member of the interleukin-6 superfamily, and the propeptide of collagen Type I (PIP) in arterial hypertension were increased and associated with LVH [Querejeta et al. 2000].

In a cross-sectional study it was demonstrated that fetuin-A deficiency is associated with inflammation and links vascular calcification to mortality in patients on dialysis [Ketteler et al. 2003]. Therefore, since LVH is considered to be a surrogate marker of preclinical cardiovascular disease [Frohlich et al. 1992, Mancini et al. 2004], we hypothesized a linkage of left ventricular mass (LVM) with fetuin-A, and either inflammatory or fibrosis molecules in patients with Stage 3 chronic kidney disease (CKD).

Therefore, this study was aimed at evaluating whether fetuin-A plasma levels are decreased in patients with Stage $3 \mathrm{CKD}$, that is GFR $30-59 \mathrm{ml} / \mathrm{min}$ [K/DOQI 2002], and whether they are linked to plasma concentration of high sensitivity CRP, CT- 1 , TNF- $\alpha$, PIP and to LVM.

Moderate CKD patients with arterial hypertension were compared with a group of essential hypertensives (EH) matched for age, blood pressures and duration of hypertensive disease, and having normal renal function.

\section{Materials and methods}

In accordance with the Declaration of Helsinki and institutional guidelines, the protocol was approved by the local Ethical Committee and subjects were aware of the investigational nature of the study and agreed to participate after informed consent.

\section{Study population}

We examined 64 CKD hypertensive patients entering the program; patients were moderate CKD [K/DOQI 2002] and were defined hypertensives when clinic systolic/diastolic blood pressure was $>140 / 90 \mathrm{mmHg}$, and severity of hypertension was defined according to the Seventh Report of the Joint National Committee on Detection, Evaluation and Treatment of High Blood Pressure [Joint National Committee on Detection, Evaluation and Treatment of High Blood Pressure 2003].

Clinic blood pressure was considered as the average of three consecutive measurements using a mercury sphygmomanometer after the subject had been sitting for 5 minutes.

55 established hypertensive (EH) subjects, matched for age, body mass index (BMI), blood pressures and duration of hypertension, and with normal renal function were enrolled as controls.

In EH secondary and/or complicated forms of hypertension were ruled out by clinical examination, determination of serum creatinine, serum and urinary electrolytes, plasma catecholamine levels and renin activity, renal echography.

Glomerular filtration rate was estimated by the Cockroft-Gault formula [Cockroft and Gault 1976] in all subjects.

Exclusion criteria were age $<30$ years and $>65$ years, diabetes mellitus Type 1 or 2 previously diagnosed, secondary or accelerated-malignant arterial hypertension, history of either stroke or TIA, and of coronary heart disease or myocardial infarction, abnormalities of cardiac rhythm or conduction under pharmacological treatment, cardiac failure.

Due to ethical reasons antihypertensive medication was not withdrawn. All patients took the same classes of drugs that were carvedilol, furosemide and angiotensin receptor blockers or ACE inhibitors.

\section{Study protocol}

On the day of the study, at 9:00 a.m., with the patients in supine position and after fasting overnight, blood samples were obtained from an indwelling forearm venous catheter to assay plasma levels of fetuin-A, CRP, TNF $\alpha$, CT-1 and PIP. 
Table 1. Demographic data of patients with chronic kidney disease (CKD) and with established hypertension $(\mathrm{EH})$.

\begin{tabular}{|c|c|c|c|}
\hline & $\operatorname{CKD}(n=64)$ & $E H(n=55)$ & $p<$ \\
\hline Age years & $57 \pm 16$ & $50 \pm 12$ & ns \\
\hline Male/female & $36 / 28$ & $31 / 24$ & ns \\
\hline Duration hypertension years & $5 \pm 1$ & $5 \pm 0.8$ & ns \\
\hline $\mathrm{BMI} \mathrm{kg} / \mathrm{m}^{2}$ & $29 \pm 4.3$ & $29.5 \pm 5$ & ns \\
\hline $\mathrm{sCr} \mathrm{mg} / \mathrm{dl}$ & $2.8 \pm 1.7$ & $0.95 \pm 0.13$ & 0.001 \\
\hline GFR $\mathrm{ml} / \mathrm{min}$ & $41 \pm 9.84$ & $100.9 \pm 9.4$ & 0.001 \\
\hline PTH pg/ml & $181 \pm 117$ & $38.6 \pm 8$ & 0.001 \\
\hline $\mathrm{sCa} \mathrm{mg} / \mathrm{l}$ & $9.05 \pm 0.37$ & $10.04 \pm 0.5$ & 0.001 \\
\hline $\mathrm{sP} \mathrm{mg} / \mathrm{l}$ & $4.06 \pm 0.99$ & $3.4 \pm 0.06$ & 0.001 \\
\hline $\mathrm{Hb} \mathrm{g} / \mathrm{dl}$ & $11.3 \pm 0.9$ & $14.1 \pm 0.04$ & 0.001 \\
\hline SBP $\mathrm{mmHg}$ & $146 \pm 7$ & $146 \pm 5.7$ & ns \\
\hline $\mathrm{DBP} \mathrm{mmHg}$ & $89 \pm 8$ & $90 \pm 4$ & ns \\
\hline LMV g & $267 \pm 103$ & $182 \pm 25$ & 0.001 \\
\hline LVMI $\mathrm{g} / \mathrm{m}^{2}$ & $127 \pm 40$ & $105 \pm 25$ & 0.001 \\
\hline LVMH g/m 2.7 & $57 \pm 17$ & $50 \pm 11$ & 0.01 \\
\hline
\end{tabular}

$\mathrm{BMI}=$ body mass index, $\mathrm{sCr}=$ serum creatinine, $\mathrm{GFR}=$ glomerular filtration rate, $\mathrm{PTH}=$ partiroid hormone, $\mathrm{sCa}=$ serum calcium, $\mathrm{sP}=$ serum phosphorus, $\mathrm{SBP}=$ systolic blood pressure, DBP = diastolic blood pressure, LVM = left ventricular mass, $\mathrm{LVMI}$ left ventricular mass index, $\mathrm{LVMH}^{2.7}=$ left ventricular mass indexed by height ${ }^{2.7}$.
$10 \mathrm{ng} / \mathrm{ml}$, inter-assay coefficient of precision was $\leq 6.3 \%$.

CT-1 was assayed by Biovendor $\mathrm{GmbH}$ commercial kit (Heidelberg, Germany). The sensitivity was $1 \mathrm{pg} / \mathrm{ml}$, inter-assay coefficient of variation was $7.8 \%$ and the intra-assay coefficient of variation was $<9 \%$.

Echocardiography was performed using an Acuson Sequoia 512 system (Siemens, Mountain View, CA, USA). Images were taken in left lateral decubitus position.

Only those frames with optimal visualization of interfaces and showing simultaneous visualization of septum, left ventricular diameters and posterior wall were used for readings.

Left ventricular mass (LVM) was determined using the ASE-corrected cube formula [Devereux et al. 1986] and was indexed by body surface area (LVMI) and by height elevated by a power of 2.7, as suggested by De Simone et al. [1992] (LVMH $\left.{ }^{2.7}\right)$.

Echocardiographic data are expressed as the average of 5 consecutive cardiac cycles. Images were read by a single cardiologist, who was blinded to the patient's clinical characteristics.

\section{Statistical methods}

Fetuin-A, hs-CRP, TNF- $\alpha$, CT-1, and PIP were measured by ELISA using a solid-phase specific sandwich enzyme-linked immunosorbent assay. Standard curves were constructed using appropriated concentrations for each factor. Precautions were taken to avoid interference with other serum components [Deanfield et al. 2005].

Fetuin-A was measured by commercial kit (Epitope Diagnostics Inc., San Diego, CA, USA), the sensitivity was $2.5 \mathrm{ng} / \mathrm{ml}$.

High sensitivity CRP was measured by commercially available ELISA kit (Diagnostic Biochem, London, Canada). The sensitivity was $10 \mathrm{ng} / \mathrm{ml}$, inter-assay coefficient of variation was $<10 \%$ and the intra-assay coefficient of variation was $<8 \%$.

Tumor necrosis factor- $\alpha$ was assayed by Amersham Biosciences kit (Little Chalfont, England), sensitivity was $<5 \mathrm{pg} / \mathrm{ml}$, reproducibility of both intra- and between-assay had a coefficient of variation $<10 \%$.

PIP was analyzed by Takara Bio Inc. commercial kit (Otsu, Japan). The sensitivity was
Differences between groups were evaluated using ANOVA and Student's t-test. Simple and multiple regression analyses were used to test the relationships between the variables.

The multiple regression analysis was carried out both in the two groups of subjects and in the group resulting from the association of CKD with EH patients $(n=119)$.

These analyses considered LVMI (or $\mathrm{LVMH}^{2.7}$ ) as a dependent variable, in a model comprising age, blood pressures, BMI, lipid variables, GFR, Hb, PTH, fetuin-A, hs-CRP, PIP, CT-1, TNF $\alpha$ and anti-hypertensive treatment (coded as follows: 0: no treatment, 1 : previous treatment, 2: ACEIs or ARBs, 3: diuretics, 4: $\beta$-blockers, 5 : combination of 2 or more drugs). To avoid the problem of spurious association deriving from multiple correlations only associations with $\mathrm{p}<0.001$ were considered as statistically significant. Results are given as means $\pm \mathrm{SD}$. The null hypothesis was rejected at a 2 -tailed $\mathrm{p}<0.05$. The statis- 
Table 2. Mean levels of plasma concentrations of fetuin-A, Tumor necrosis factor (TNF), high sensitivity C-reactive Protein (hs-CRP), Cardiotrophin-1 (CT-1) and propeptide of collagen type 1 (PIP) in 64 patients with chronic kidney disease (CKD) and in 55 established essential hypertensives (EH) with and without left ventricular hypertrophy (LVH).

\begin{tabular}{|c|c|c|c|c|}
\hline & \multicolumn{2}{|c|}{ Chronic Kidney Disease } & \multicolumn{2}{|c|}{ Essential Hypertension } \\
\hline & $\begin{array}{l}\text { without LVH } \\
\qquad n=34\end{array}$ & $\begin{array}{c}\text { with LVH } \\
n=30\end{array}$ & $\begin{array}{l}\text { without LVH } \\
\qquad n=41\end{array}$ & $\begin{array}{c}\text { with LVH } \\
n=14\end{array}$ \\
\hline Fetuin-A g/l & $0.64 \pm 0.24^{\#}$ & $0.49 \pm 0.2^{\circ \circ *}$ & $0.96 \pm 0.18$ & $0.80 \pm 0.2$ \\
\hline $\mathrm{PIP} \mathrm{ng} / \mathrm{ml}$ & $66 \pm 29^{\#}$ & $89 \pm 40^{\circ}$ & $47 \pm 18$ & $81.7 \pm 28 \bullet \bullet \bullet$ \\
\hline CT-1 pg/ml & $63 \pm 26^{\#}$ & $87 \pm 31^{0000}$ & $38.5 \pm 25$ & $73.4 \pm 34^{\bullet \bullet \bullet}$ \\
\hline hs-CRP mg/dl & $3.28 \pm 0.9^{\#}$ & $3.91 \pm 0.9^{\circ 00 *}$ & $1.9 \pm 0.6$ & $2.4 \pm 0.5^{\bullet \bullet}$ \\
\hline $\mathrm{TNF} \alpha \mathrm{ng} / \mathrm{ml}$ & $4.6 \pm 0.77^{\#}$ & $5 \pm 0.95^{\star}$ & $3.2 \pm 0.5$ & $3.9 \pm 0.25^{\bullet \bullet \bullet}$ \\
\hline LVMI $\mathrm{g} / \mathrm{m}^{2}$ & $92.5 \pm 12$ & $138.8 \pm 18^{\circ 00 \circ}$ & $93 \pm 14$ & $143 \pm 16^{\bullet \bullet \bullet}$ \\
\hline
\end{tabular}

${ }^{*} p<0.001$ CKD vs. EH with LVH, ${ }^{\#} p<0.001$ CKD vs. EH without LVH, ${ }^{\circ} p<0.01,{ }^{\circ} p<0.009,{ }^{\circ 00} p<0.007$, ${ }^{\circ 000} p<0.001$ CKD with LVH vs. CKD without LVH, ${ }^{\bullet} p<0.007,{ }^{\bullet \bullet} p<0.0001 \mathrm{EH}$ with LVH vs. EH without $\mathrm{LVH}$
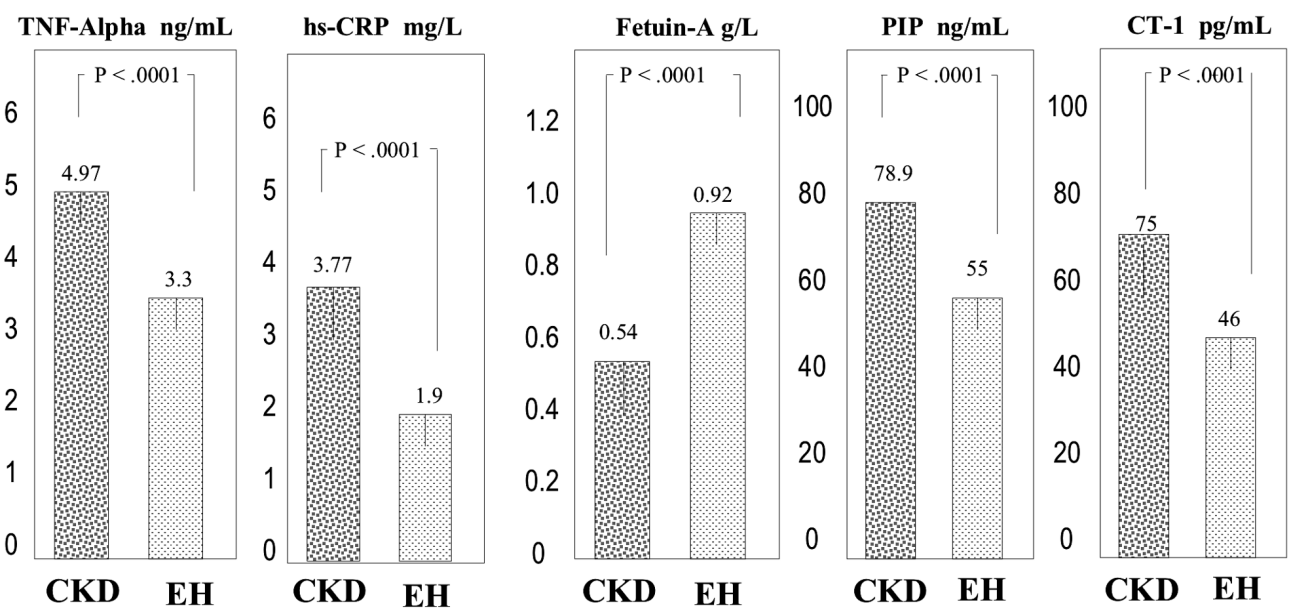

Figure 1. Mean levels of plasma concentrations of Fetuin-A, Tumor Necrosis Factor (TNF), high sensitivity $\mathrm{C}$ - reactive protein (hs-CRP), Cardiotrophin-1 (CT-1) and propeptide of collagen type I (PIP) in 64 patients with chronic kidney disease (CKD) and in 55 established essential hypertensives (EH)

tical analyses were performed using the SYSTAT DATA software package, version 5.2 (Systat, Evanston, IL, USA).

\section{Results}

Table 1 gives demographic data of CKD and EH patients. The comparison between hypertensive $\mathrm{CKD}$ and hypertensive controls showed quite similar values of age, BMI, blood pressures and duration of hypertensive disease.

Fetuin-A plasma levels were significantly lower in hypertensive CKD than in EH patients (Figure 1), this difference was statistically significant even after adjustment for age $(p=0.001)$ and for both age and CRP $(p=$ 0.01 ). Plasma concentration of hs-CRP as well as of TNF- $\alpha$, PIP, and CT-1 were higher in CKD than in EH (Figure 1).

Among CKD there were 30/64 patients with left ventricular hypertrophy (46.0\%), and in EH group 14/55 (25.5\%) subjects had LVH.

The comparison between moderate CKD having LVH (n 30) with those without LVH (n 34) showed significant differences in plasma levels of fetuin-A ( $p<0.002)$, TNF- $\alpha(\mathrm{p}<$ $0.01)$ and hs-CRP $(\mathrm{p}<0.001)$, CT- 1 and PIP $(p<0.002)$ (Table 2). No significant differences in estimated GFR between the two subgroups were observed ( $44 \pm 6.8$ vs $40 \pm 8.9$ $\mathrm{ml} / \mathrm{min}, \mathrm{NS}$ ). 


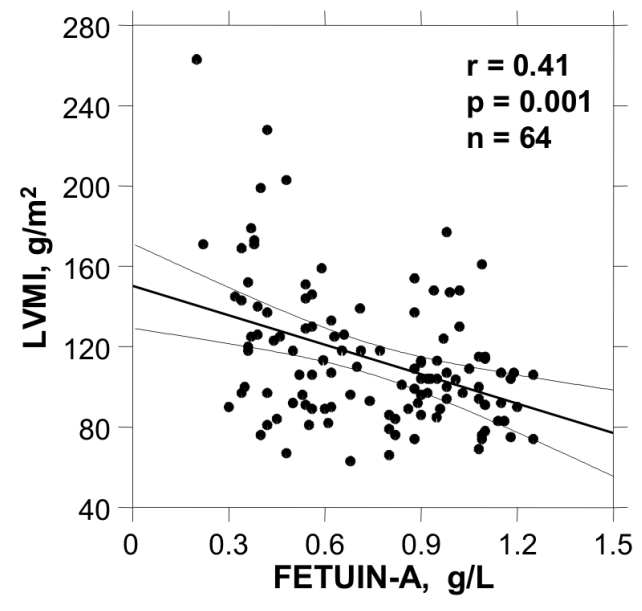

Figure 2. Correlation of Fetuin-A with Left Ventricular Mass Index (LVMI) in moderate chronic kidney disease patients.

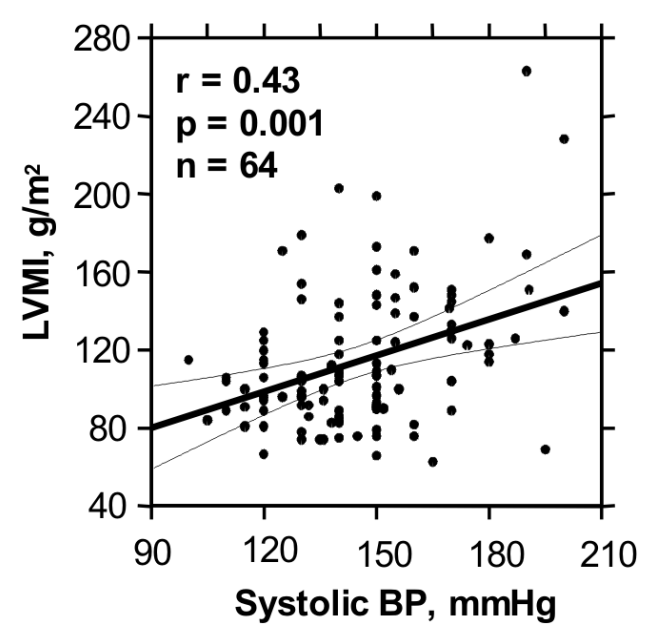

Figure 3. Correlation of Systolic Blood Pressure (SBP) with Left Ventricular Mass Index (LVMI) in moderate chronic kidney disease patients.

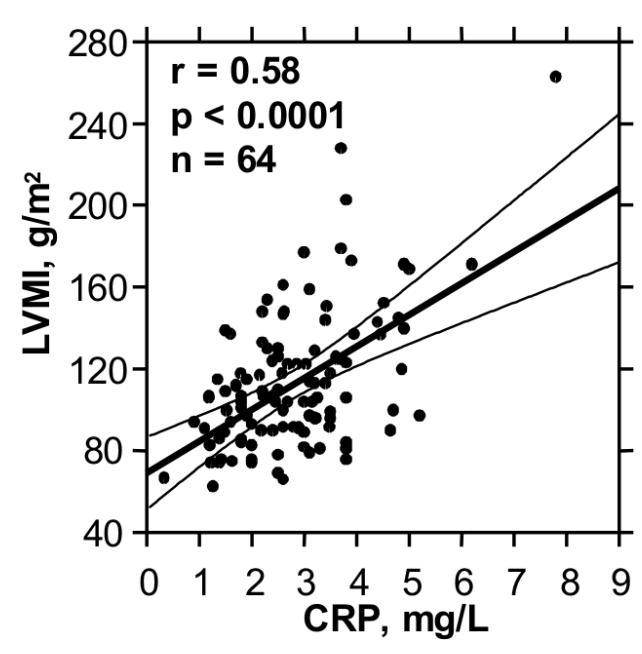

Figure 4. Correlation of $\mathrm{C}$ - reactive protein (CRP) with Left Ventricular Mass Index (LVMI) in moderate chronic kidney disease patients.
On the contrary, we did not observe any difference among fetuin-A levels when comparing EH having and not-having $\mathrm{LVH}$, whereas significant differences between the other molecules were found (Table 2).

A further comparison between CKD and EH patients with $\mathrm{LVH}$ demonstrated lower values of fetuin-A $(0.49 \pm 0.2$ vs $0.80 \pm 0.2$ $\mathrm{g} / \mathrm{l}, \mathrm{p}<0.001)$, and higher values of hs-CRP $(3.91 \pm 0.9$ vs $2.4 \pm 0.5 \mathrm{mg} / \mathrm{l}, \mathrm{p}<0.001)$, TNF- $\alpha(5.25 \pm 1.24$ vs $3.5 \pm 0.51 \mathrm{ng} / \mathrm{ml}$, $\mathrm{p}<0.001)$, CT-1 (87 \pm 31 vs $73.4 \pm 34 \mathrm{pg} / \mathrm{ml}$, $\mathrm{p}<0.001)$ and PIP $(89 \pm 40$ vs $81.7 \pm 28 \mathrm{ng} /$ $\mathrm{ml}, \mathrm{p}<0.001$ ) in CKD than in EH (Table 2).

\section{Univariate and multivariate analyses of correlation}

In CKD patients the univariate analysis of correlation demonstrated that fetuin-A was inversely correlated with serum creatinine $(\mathrm{r}-0.70, \mathrm{p}<0.001)$, plasma phosphorus levels $(\mathrm{r}-0.47, \mathrm{p}<0.001)$, and PTH concentrations $(\mathrm{r}-0.557, \mathrm{p}<0.001)$. Estimated GFR also correlated with fetuin-A ( $\mathrm{r} 0.78, \mathrm{p}<0.001)$.

Furthermore, inverse and significant correlations of fetuin-A with TNF- $\alpha(\mathrm{r}-0.714$, $\mathrm{p}<0.001)$, and hs-CRP $(\mathrm{r}-0.65, \mathrm{p}<0.001)$ plasma levels were found. Interestingly, in CKD patients fetuin-A correlated negatively to LVMI ( $\mathrm{r}-0.41, \mathrm{p}=0.001)$ (Figure 2). In turn, LVMI showed to be directly correlated to systolic blood pressure ( $\mathrm{r} 0.43, \mathrm{p}=0.001$ ) (Figure 3), and to hs-CRP (r 0.58, p < 0.0001 ) (Figure 4). On the contrary, in EH controls no correlation between fetuin-A and LVMI was found ( $\mathrm{r}-0.07$, NS), whereas LVMI was significantly correlated to hs-CRP ( $\mathrm{r} 0.44, \mathrm{p}<$ $0.0008)$. In both groups no significant correlation between fetuin-A and, respectively, BMI or lipid values was found.

The multiple regression analysis was carried out considering LVMI (or $\mathrm{LVMH}^{2.7}$ ) as a dependent variable, in a model comprising age, blood pressures, BMI, lipid variables, GFR, Hb, PTH, fetuin-A, hs-CRP, PIP, CT-1, TNF $\alpha$ and anti-hypertensive treatment.

This analysis demonstrated that in CKD patients hs-CRP $(\beta 0.64, \mathrm{p}<0.004)$, and systolic blood pressure $(\beta 0.32, \mathrm{p}<0.006)$ were the independent predictors of LVMI. The relationship between LVMI and fetuin-A did not maintain statistical significance $(\beta-0.27$, 
NS). Similar results were obtained when left ventricular mass was indexed by height elevated by a power of 2.7 , being this latter independently associated with hs-CRP ( $\beta 0.60$, $\mathrm{p}<0.004)$, systolic blood pressure $(\beta 0.31$, $\mathrm{p}<0.006)$ and BMI $(\beta 0.39, \mathrm{p}<0.0004)$, but not with fetuin-A $(\beta-0.26$, NS).

The same multiple regression model applied to the overall study population $(n=119)$ confirmed the results observed in the patients with CKD. In fact, the independent correlates of LVMI were hs-CRP $(\beta 0.48, \mathrm{p}<0.00001)$, and systolic blood pressure $(\beta 0.27, \mathrm{p}<0.001)$, and those of $\mathrm{LVMH}^{2.7}$ were hs-CRP $(\beta 0.47$, $\mathrm{p}<0.00001)$, systolic blood pressure $(\beta 0.24$, $\mathrm{p}<0.003)$ and BMI $(\beta 0.26, \mathrm{p}<0.0001)$.

\section{Discussion}

The development of LVH is influenced by a complex interplay of hemodynamic, neurohumoral, genetic and environmental determinants [Frohlich et al. 1992].

LVH is the most common cardiac complication caused by hypertension, and is a major risk factor for overall and cardiovascular mortality in patients with end-stage renal disease [Levy et al. 1990]. However, limited data exist on determinants of $\mathrm{LVH}$ in patients in the earliest stages of CKD [Astor et al. 2004], and to the best of our knowledge, this is the first report regarding the relationship of left ventricular mass with hs-CRP and fetuin-A in patients with moderate CKD.

One most relevant datum of our study is given by the demonstration of decreased plasma levels of fetuin-A, associated with increased hs-CRP, and LVMI in moderate CKD patients in comparison with essential hypertensives having normal renal function, similar BMI, and matched for blood pressures and duration of hypertensive disease. We previously demonstrated that hs-CRP and TNF- $\alpha$ are increased in $\mathrm{EH}$ when compared with healthy normotensive subjects [Cottone et al. 2006], and that in non-uremic CKD plasma levels of TNF- $\alpha$ are higher than in $\mathrm{EH}$ [Cottone et al. 1998, 2002].

In this regard, the results of the present study are in line with our previous results and with those by other studies carried out in both patients not undergoing dialysis and undergo- ing dialysis [Shlipak et al. 2003, Stam et al. 2003, Wang et al. 2004].

Most noteworthy is the evidence that even in moderate CKD patients plasma levels of fetuin-A are significantly decreased, and associated inversely with both LVM and hs-CRP. These data are strengthened by the observation of levels of fetuin-A even lower in CKD patients having LVH than in those not-having LVH.

Nonetheless, according to our statistical analysis, it seems that fetuin-A is not endowed with the property to induce directly LVH, but it needs CRP activation. Although fetuin-A was no longer statistically significant at entry of CRP into the multivariate regression analysis, its dependence on CRP suggests a biologically relevant role in the development or maintenance of inflammation and myocardial changes.

Even if similar data were observed in dialyzed patients [Ketteler et al. 2003], this is just a hypothesis because it is to be established whether a statistical analysis mirrors exactly a biologic phenomenon.

In a cross-sectional study it was demonstrated that fetuin-A deficiency is associated with inflammation and links vascular calcification to mortality in patients on dialysis [Ketteler et al. 2003]. Low concentrations of the glycoprotein were associated with raised amounts of CRP and with enhanced cardiovascular and all-cause mortality. Nevertheless, very recently, it has been reported [Ix et al. 2006] in a cohort of persons with coronary artery disease and across a broad range of GFR, that fetuin-A was not inversely associated with renal function, estimated by four different methods.

In contrast with the data and the conclusions by Ix et al. [2006], our results seem to show that both reduction of fetuin-A and its linkage with CRP and cardiac complication take place long before the uremic condition appears.

Actually, between our study and that by Ix et al. [2006] there are several marked differences.

First of all, our study was aimed at evaluating fetuin-A in patients having $\mathrm{LVH}$, not coronary calcification, we analyzed GFR only by one estimation method, and in a smaller group even considering in the study by Ix just that subgroup with estimated GFR more similar to that of our $64 \mathrm{CKD}$ patients. 
Finally, when Ix and colleagues examined the likelihood of decreased fetuin-A concentrations, they found a reduced odds of low fetuin-A among subjects in the higher quartiles of cystatin-C. These data suggest that the estimation method of GFR could influence the results.

Based on an experimental study it was hypothesized that in fetuin-A-deficient mice the severe diastolic dysfunction observed was ventricular remodeling triggered by a combination of both cardiac calcification and fibrosis [Merx et al. 2005].

Serum markers of collagen synthesis or degradation have been investigated and found to be altered in hypertension [Gonzales et al. 2005, Querejeta et al. 2000]. The carboxyterminal propeptide of procollagen Type I (PIP) correlated with collagen volume fraction on endomyocardial biopsies [Querejeta et al. 2000].

In our study, PIP was significantly higher in patients having $\mathrm{LVH}$, both in $\mathrm{CKD}$ and $\mathrm{EH}$ groups.

This seems to demonstrate that the increased plasma concentrations of PIP are not influenced by differences in its clearance.

In our study, the multivariate analyses confirmed that hs-CRP and systolic blood pressure are predictors of LVH even in moderate CKD patients.

The relationship between blood pressure and LVM was clearly established [Verdecchia et al. 1990]. Less clear are the mechanisms underlying the relationship of CRP with LVH.

In the Cardiovascular Health Study [Shlipak et al. 2003] renal insufficiency was independently associated with elevation of CRP, and Panichi et al. [2002] demonstrated that CRP rises as renal function declines. On the contrary, in our study, hs-CRP predicted LVMI independently of renal function.

One hypothesis could be that blood pressure may modulate the association between inflammatory activity, LVH, and renal function.

It is to note that our CKD and $\mathrm{EH}$ patients were matched for blood pressure levels and duration of hypertensive disease, and they took the same antihypertensive drugs. In spite of this, we observed higher hs-CRP levels and a greater prevalence of LVH in CKD than in EH patients.

Because of the established relation of endothelial function and cardiovascular hyper- trophy [Cottone et al. 1998, Perticone et al. 1999], a second hypothesis could consider the relationship between CRP and the nitric oxide pathway [Verma et al. 2002, Zoccali et al. 2000, 2002].

The present study has a few limitations. First of all, this is a cross-sectional, observational study. Moreover, the blood concentrations of the molecules we studied could not exactly reflect the tissue condition. Finally, it is conceivable that in renal insufficiency a reduced clearance of these molecules could take place, influencing the results.

In conclusion, in a limited fashion with the findings by our cross-sectional study, we demonstrate for the first time in moderate CKD patients that fetuin-A is decreased and related to left ventricular hypertrophy not directly, and that its biologic role depends on C-reactive protein.

This observation could support future therapeutic choices favoring anti-inflammatory treatment in an early stage of chronic kidney disease, in order to reduce cardiovascular morbility.

\section{Conflict of interest}

None.

\section{Acknowledgement}

This work was supported in part by a grant from the Italian Ministry for University and Scientific Research (MURST).

\section{References}

Astor BC et al. Association of kidney function with left ventricular morphology among African Americans: The Atherosclerosis Risk in Communities (ARIC) Study. Am J Kidney Dis. 2004; 43: 836-845.

Blake GJ et al. Blood pressure, C-reactive protein, and risk of future cardiovascular events. Circulation. 2003; 108: 2993-2999.

Chertow GM et al. Determinants of progressive vascular calcification in hemodialysis patients. Nephrol Dial Transplant. 2004; 19: 1489-1496.

Clausell $N$ et al. Increased expression of tumor necrosis factor- $\alpha$ in diabetic macrovasculopathy. Cardiovasc Pathol. 1999; 8: 145-151.

Cockroft DW, Gault MH. Prediction of creatinine clearance from serum creatinine. Nephron. 1976; 16 : $31-41$. 
Cottone $S$ et al. Changes of plasma endothelin and growth factor levels, and of left ventricular mass, after chronic AT1-receptor blockade in human hypertension. Am J Hypertens. 1998; 11: 548-553.

Cottone $S$ et al. Comparison of tumour necrosis factor and endothelin-1 between essential and renal hypertensive patients. J Hum Hypertens. 1998; 12: 351 354.

Cottone $S$ et al. Amplified biochemical activation of endothelial function in hypertension associated with moderate to severe renal failure. J Nephrol. 2002; 15: 643-648.

Cottone $S$ et al. Relation of C-reactive protein to oxidative stress and to endothelial activation in essential hypertension. Am J Hypertens. 2006; 19: 313-318.

Deanfield $J$ et al. Working Group on Endothelin and Endothelial Factors of the European Society of Hypertension: Endothelial function and dysfunction. Part I: methodological issues for assessment in the different vascular beds: a statement by the Working Group on endothelin and endothelial factors of the European Society of Hypertension. J Hypertens. 2005; 23: 7-17.

De Simone $G$ et al. Left ventricular mass and body size in normotensive children and adults: assessment of allometric relations and the impact of overweight. J Am Coll Cardiol. 1992; 20: 1251-1260.

Devereux RB et al. Echocardiographic assessment of left ventricular hypertrophy: comparison to necropsy findings. Am J Cardiol. 1986; 57: 450-458.

Feldman AM et al. The role of tumor necrosis factor in the pathophysiology of heart failure. J Am Coll Cardiol. 2000; 35: 537-544.

Frohlich ED et al. The heart in hypertension. N Engl J Med. 1992; 327: 998-1008.

Gonzales A et al. Usefulness of plasma cardiotrophin-1 in assessment of left ventricular hypertrophy regression in hypertensive patients. J Hypertens. 2005; 23 . 2297-2304

Ix JH et al. Fetuin-A and kidney function in persons with coronary artery disease - data from the heart and soul study. Nephrol Dial Transplant. 2006; 21:2144-2151

Joint National Committee on Detection, Evaluation and Treatment of High Blood Pressure. The Seventh Report of The Joint National Committee, Evaluation and Treatment of High Blood Pressure. JAMA. 2003; 157 : 2413-2446.

$K / D O Q I$. Clinical practice guidelines for chronic kidney disease: evaluation, classification, and stratification. Am J Kidney Dis. 2002; 39 (Suppl 1): S1-S266.

Ketteler $M$ et al. Association of low fetuin-A (AHSG) concentrations in serum with cardiovascular mortality in patients on dialysis: a cross-sectional study. Lancet. $2003 ; 361: 827-833$

Levy $D$ et al. Prognostic implications of echocardiographically determined left ventricular mass in the Framingham Heart Study. N Engl J Med. 1990; 322: 1561-1566.

Mancini GBJ et al. Surrogate markers for cardiovascular disease: structural markers. Circulation. 2004; 109 (Suppl 1): IV22- IV30.

Merx MW et al. Myocardial stiffness, cardiac remodelling, and diastolic dysfunction in calcification-prone fetuin-A-deficient mice. J Am Soc Nephrol. 2005; 16 : 3357-3364.

Panichi V et al. C-reactive protein and interleukin-6 levels are related to renal function in predialytic chronic renal failure. Nephron. 2002; 91: 594-600.
Perticone $F$ et al. Relationship between left ventricular mass and endothelium-dependent vasodilation in never-treated hypertensive patients. Circulation. 1999; 99: 1991-1996.

Querejeta R et al. Serum carboxy-terminal propeptide of procollagen Type I is a marker of myocardial fibrosis in hypertensive heart disease. Circulation. 2000; 101 . 1729-1735.

Ross $R$. Atherosclerosis - an inflammatory disease. N Engl J Med. 1999; 340: 115-126.

Shlipak $M G$ et al. Elevations of inflammatory and procoagulant biomarkers in elderly persons with renal insufficiency. Circulation. 2003; 107: 87-92.

Sladek $T$ et al. Morphometric characteristics of cardiac hypertrophy induced by long-term inhibition of $\mathrm{NO}$ synthase. Physiol Res. 1996; 45: 335-338.

Stam $F$ et al. Impaired renal function is associated with markers of endothelial dysfunction and increased inflammatory activity. Nephrol Dial Transplant. 2003; 18: 892-898.

Verdecchia P et al. Circadian blood pressure changes and left ventricular hypertrophy in essential hypertension. Circulation. 1990; 81: 528-536.

Verma $S$ et al. A self-fulfilling prophecy. C-reactive protein attenuates nitric oxide production and inhibits angiogenesis. Circulation. 2002; 106: 913-919.

Waltenberger J et al. C-reactive protein frequently colocalizes with the terminal complement complex in the intima of early atherosclerotic lesions of human coronary arteries. Arterioscler Thromb Vasc Biol. 1998; 18: 1386-1392.

Wang AYM et al. Inflammation, residual kidney function, and cardiac hypertrophy are interrelated and combine adversely to enhance mortality and cardiovascular death risk of peritoneal dialysis patients. J Am Soc Nephrol. 2004; 15: 2186-2194.

Zoccali $C$ et al. Inflammation is associated with carotid atherosclerosis in dialysis patients. J Hypertens. 2000; 18: 1207-1213.

Zoccali $C$ et al. Asymmetric dimethylarginine, C-reactive protein, and carotid-intima media thickness in end-stage renal disease. J Am Soc Nephrol. 2002; 13. 490-496. 
Copyright of Clinical Nephrology is the property of Dustri-Verlag Dr. Karl Feistle GmbH \& Co., $K G$ and its content may not be copied or emailed to multiple sites or posted to a listserv without the copyright holder's express written permission. However, users may print, download, or email articles for individual use. 